\title{
Image Processing for Automated Flaw Detection and CMYK model for Color Image Segmentation using Type 2 Fuzzy Sets
}

\author{
Anindita Chatterjee, Himadri Nath Moulick \\ (Dept-IT, Tata Consultancy Services Kolkata, India) \\ (Dept-CSE, Aryabhatta Institute of Engg \& Management Durgapur, pin-713148, India)
}

\begin{abstract}
Infrared (IR) thermography has evolved in recent years from being an emerging nondestructive testing (NDT) technique to a viable approach for both aerospace manufacturing and in-service inspections. One of the drawbacks of thermography techniques is that no standard signal processing has been universally adopted and that different algorithms yield different sizing results. Additionally, the data interpretation is not as simple as with other NDT techniques. In this paper the most common signal processing techniques applied to pulsed thermography, which include derivative processing, pulsed phase thermography and principal component analysis, are applied in an attempt to simplify the damage detection and sizing process. The pulsed thermography experiments were carried out on 25 impacted panels made of carbon fiber epoxy material. Despite using similar panels and the same experiment parameters, the damage detection and sizing processes are not straight forward. It is concluded that some algorithms provide easier detection capability than others. However, on their own, the different algorithms lack the robustness to make the damage detection and sizing processes reliable and fully automated. And Optimization of the similarity measure is an essential theme in medical image registration. In this paper, a novel continuous medical image registration approach (CMIR) is proposed. This is our extension work of the previous one where we did a segmentation part of any particular image with a custom algorithm. The CMIR, considering the feedback from users and their preferences on the trade-off between global registration and local registration, extracts the concerned region by user interaction and continuously optimizing the registration result. Experiment results show that CMIR is robust, and more effective compared with the basic optimization algorithm. Image registration, as a precondition of image fusion, has been a critical technique in clinical diagnosis. It can be classified into global registration and local registration. Global registration is used most frequently, which could give a good approximation in most cases and do not need to determine many parameters. Local registration can give detailed information about the concerned regions, which is the critical region in the image. Finding the maximum of the similarity measure is an essential problem in medical image registration. Our work is concentrating on that particular section with the synergy of Tpe-2 fuzzy logic invoked in it.
\end{abstract}

Keywords; Extrinsic method, Fuzzy sets, image possessing, intrinsic method Introduction, Multi-model image alignment.

\section{INTRODUCTION}

In pulsed thermography (PT) energy is applied to the specimen using a pulsed excitation. Typically, the energy sources are flash lamps whose flash duration varies from a few milliseconds for good thermal conductors to a few seconds for low-conductivity materials. The applied energy creates a thermal front that propagates from the specimen's surface throughout the specimen. During the cool down process the surface temperature decreases uniformly for a sample without internal flaws. When the thermal front intersects an interface from a high to low conductivity layer, like in the case of delamination, disband and porosity, the cooling rate is locally disrupted. This results in an accumulation of heat above the flaw that is also manifested at the specimen's surface and can be detected by an IR camera. Thus, allowing defective areas to be distinguished from sound areas. Image processing is commonly used for two purposes. Its first use is to improve the visual appearance of images to a human viewer. Filtering and color map adjustments are commonly applied to make an image more pleasant to look at. Its second purpose is to prepare the images or data for the measurement of features present. This can include applying a threshold to create a binary image, applying morphologic filters, etc. The processed image allows the operator to measure the size of the features of interest and could also be used for automated flaw detection and measurements. Although NDT inspections are more and more automated, thanks to automated scanning systems, the inspector is still required to identify the presence of flaw and to perform the measurement of the features of interest. The following paragraphs review the most common signal processing techniques applied to pulsed thermography data. In the open literature these algorithms are usually applied and demonstrated on laboratory samples that contain artificial and simple geometry flaws. In this paper, solid laminate samples that have been damaged by impact are used to investigate the capability of these algorithms for the development of robust and automated flaw detection and measurements. And Information systems are 
often poorly defined, creating difficulty in representing concepts and selecting important features used to solve the problems. Type-1 (T1) fuzzy set (FS) has been around for more than four decades and yet not able to handle all kinds of uncertainties appearing in real life. The above statement sounds paradoxical because the word fuzzy has the connotation of uncertainty. The extension of T1 fuzzy systems, in particular type-2 (T2) accommodates the system uncertainties and minimizes its effect considerably in decision making. However, T2 FS is difficult to understand and explain. Application of T1 fuzzy logic to rule-based systems is most significant that demonstrates its importance as a powerful design methodology to tackle uncertainties. A fuzzy logic system (FLS) is described completely in terms of T1 fuzzy sets, called type-1 fuzzy logic system (T1FLS), whereas a FLS with at least one T2 fuzzy set is called T2 fuzzy logic system (T2FLS). T1FLSs cannot directly handle rule uncertainties because T1 fuzzy sets are certain. On the other hand, T2FLS is very useful in circumstances where it is difficult to determine an exact membership function of a fuzzy set. Such cases are handled by rule uncertainties and measurement uncertainties. Like T1FLS, T2 has wide applications and the potential of T2 systems outperforms T1 in most of the cases. The aim of the paper is to describe T2 fuzzy systems for managing uncertainties, identifying the frontier research areas where T2 fuzzy logic is applied and proposes an algorithm on application of type-2 fuzzy sets in color image segmentation.

\section{THERMAL CONTRASTS}

The most basic data processing performed on pulsed thermographic data is the computation of thermal contrasts. Thermal contrasts have the advantages of being less sensitive to noise and to the surface optical properties1. The main problem with thermal contrast computation is that it requires a priori knowledge of a sound area. Although more recently new contrast methods have been developed to overcome this problem

\section{Pulsed Phase Thermography}

Pulsed phase thermography (PPT) is a processing method in which the thermal images are transformed from the time domain to the frequency domain5. This can be performed by processing a sequence of thermal images (thermogram) with discrete Fourier transform (DFT):

$$
F_{n}=\sum_{k=0}^{N-1} T(k) e^{-2 \pi i k n / N}=\mathrm{Re}_{n}+i \operatorname{Im}_{n}
$$

Where $\mathrm{n}$ designates the frequency increments $(\mathrm{n}=0,1, \ldots \mathrm{N}-1)$, and $\mathrm{Re}$ and $\mathrm{Im}$ are the real and the imaginary parts of

the DFT, respectively. For convenience, fast Fourier transform (FFT) a computationally efficient version of the DFT is generally used. Once the data has been converted into the Fourier domain, the phase ( $\square$ ) and amplitude (A) images of the different frequencies can be calculated using:

$$
\begin{gathered}
A_{n}=\sqrt{\mathrm{Re}_{n}{ }^{2}+\operatorname{Im}_{n}{ }^{2}} \\
\text { and } \\
\phi_{n}=\tan ^{-1}\left(\frac{\mathrm{Im}_{n}}{\mathrm{Re}_{n}}\right)
\end{gathered}
$$

The phase is particularly advantageous since it is less affected by environmental reflections, emissivity variations, non-uniform heating, surface geometry and orientation. The phase characteristics are very attractive not only for qualitative inspections but also for quantitative ones [6].

\section{THERMOGRAPHIC SIGNAL RECONSTRUCTION}

Thermographic signal reconstruction (TSR) 8 is a processing technique that uses polynomial interpolation to allow increasing the spatial and the temporal resolution of a thermogram sequence, while reducing the amount of data to be analyzed. TSR is based on the assumption that temperature profiles for nondefective areas follow the decay curve given by the one-dimensional solution of the Fourier diffusion equation for an ideal pulse uniformly applied to the surface of a semi-infinite body9, which is given by: 


$$
T(t)=\frac{Q}{e \sqrt{\pi \cdot t}}
$$

Where $\mathrm{T}(\mathrm{t})$ is the temperature evolution, $\mathrm{Q}$ is the energy applied at the surface and $e$ is the thermal effusively of the sample, which is defined as: $e \square \square k \square c$; where $k, \rho$, and $c$ are the thermal conductivity, the mass density and the specific heat, respectively. Equation 3 may be rewritten in a logarithmic notation and expanded into a polynomial series.

$$
\begin{aligned}
& \ln (\Delta T)=\ln \left(\frac{Q}{e}\right)-\frac{1}{2} \ln (\pi t) \\
& =a_{0}+a_{1} \ln (t)+a_{2} \ln ^{2}(t)+\ldots+a_{n} \ln ^{n}(t)
\end{aligned}
$$

The noise reduction resulting from this polynomial interpolation $[11],[12]$ enables the use of derivate processing to enhance the contrast created by the presence of defects. The first and second derivatives of the thermogram sequence provide information on the rate of temperature variation. These measurements are analogous to the relations between position, velocity and acceleration in mechanics. The original thermogram corresponds to the surface temperature of the inspected object (position). The first derivative gives information on the cooling rate of the surface temperature (velocity), while the second derivative provides information on the acceleration or deceleration of this cooling rate (acceleration).

\section{Principal COMPONENT ANALysis}

Principal component analysis (PCA) 13, also known as principal component thermography (PCT) 14, is an orthogonal linear transformation that transforms the thermogram sequence into a new coordinate system. The idea behind PCA is to remove possible correlation in the data by creating a new uncorrelated dataset called principal components. It has been applied in thermal NDT for data reduction and flaw contrast enhancement. The algorithm is based on the decomposition of the thermogram into its principal components using singular value decomposition (SVD). The first step of the PCA algorithm is to reshape the three-dimensional thermogram into a two dimensional array where the columns and rows contain the spatial and temporal information, respectively. Thus, the original thermogram $T(x, y, t)$ becomes $A(n, m)$ where $n=N x \square \square \mathrm{Ny}, \mathrm{m}=$ $\mathrm{Nt}, \mathrm{Nx}$ and $\mathrm{Ny}$ are the number of pixels per row and column of the IR camera and $\mathrm{Nt}$ is the number of thermal images in the thermogram sequence. The two-dimensional array A is then adjusted by subtracting the mean along the time dimension, and decomposed into eigenvectors and eigenvalues.

$$
\mathrm{A}=\mathrm{U} \Gamma \mathrm{V}^{\mathrm{T}}
$$

Where $\mathrm{U}$ and $\mathrm{V}$ are orthogonal matrices which columns form the eigenvectors of AAT and ATA respectively, and $\Gamma$ is a diagonal matrix that contains the singular values of ATA. Since the thermal images in the thermogram are non-erratic and vary slowly in time, the principal temporal variations of the dataset are usually contained within the first few eigenvectors. The principal component images are formed by calculating the dot product of the eigenvector and the measured temperature.

\section{MODELING UNCERTAINTY USING OF FUZZY LOGIC}

Uncertainty appears in many forms and independent of the kind of fuzzy logic (FL) or any kind of methodology one uses to handle it . Uncertainty involves in real life, due to deficiency of information in various forms. One of the best sources for general discussions about uncertainty is found in. Two types of uncertainties, randomness and fuzziness exist, where probability theory is associated with the former and FS with the latter. Fuzziness (or vagueness) generally recognizes uncertainty resulting from the imprecise boundaries of fuzzy sets, nonspecificity connected with sizes (cardinalities) of relevant sets and strife (or discord), which expresses conflicts among the various sets of alternatives. T1 fuzzy sets are certain and not able to handle all kinds of uncertainties using a single membership value, which is crisp. A FLS needs some measure to capture uncertainties than just a single number. The extended FL, named as T2FL able to handle uncertainties by modeling and subsequently minimizing their effects. T2 fuzzy logic provides the measure of dispersion, fundamental to the design of systems that includes linguistic or numerical uncertainties translating into rules. T2 fuzzy set is a natural framework for handling both randomness and fuzziness. It is the third dimension of T2 membership function (MF) that allows us to evaluate the model uncertainties. A T2FLS has more design 
degrees of freedom than a T1FLS because T2 fuzzy sets are described by more parameters compare to T1 fuzzy sets. Linguistic and random uncertainties are evaluated using the defuzzified and type-reduced outputs of the T2FLS. The type-reduced output can be interpreted as a measure of dispersion about the defuzzified output.

\section{SCOPE OF WORK}

Image segmentation is one of the most difficult image processing tasks because the segmented images are not always precise rather vague. In earlier works, image segmentation was applied in monochrome color images, later applied on red, green, blue (RGB) color space. Two main image segmentation techniques are described in the literature; region reconstruction where image plane is analyzed using region growing process and color space analysis where the color of each pixel is represented in the designated color space. Many authors have tried to determine the best color space for some specific color image segmentation problems, however, there does not exist a unique color space for all segmentation problems. Computational complexity may increase significantly with reference to C(Cyan), M(Magenta), Y(Yellow), K(contrast) (CMYK) color space in comparison with gray scale image segmentation. Classically, the RGB color space has been chosen for color image segmentation where a point in the image is defined by the color component levels of the corresponding R, $\mathrm{G}$ and $\mathrm{B}$ pixels. However, while the region growing techniques tend to over-segment the images, on the other hand the color space analysis methods are not robust enough to significance appearance changes because of not including any spatial information. Fuzzy logic is considered to be an appropriate tool for image analysis, applicable in CMYK and particularly for gray scale segmentation. Recently, fuzzy region oriented techniques and fuzzy entropy based techniques are applied for color image segmentation. The major concern of these techniques is spatial ambiguity among the pixels, representing inherent vagueness. However, there still remain some sources of uncertainties with the meanings of the words used for noisy measurements and the data used to tune the parameters of T1 fuzzy sets may be noisy too. The new concept of evidence theory allows to tackling imprecision in model uncertainty used in pattern classification, and produces good results in segmentation, although this technique based on CMYK model is not often used.

The amount of uncertainty is evaluated using the approach proposed by Klir where he generalizes the Shannon entropy to belief functions using two uncertainty measures, mainly the non-specificity and the discord. The robust method using T2 fuzzy set is another approach for handling uncertainty in image analysis. It can take into account three kinds of uncertainty, namely fuzziness, discord and nonspecificity. T2 fuzzy sets have grade of membership value, which are themselves fuzzy. Hence, the membership function of a T2 fuzzy set has three dimensions and it is the new third dimension that

\section{PRELIMINARIES OF TYPE-2 FUZZY SYSTEM}

The term "fuzzy set" is general that include T1 and T2 fuzzy sets (and even higher-type fuzzy sets). All fuzzy sets are characterized by MFs. A T1 fuzzy set is characterized by a two-dimensional MF, whereas a T2 fuzzy set is characterized by a three-dimensional MF. Let us take an example of linguistic variable "speed". Different values of the variable like "very high speed", "high speed", "low speed" signify the crisp value. One approach to using the 100 sets of two endpoints is to average the endpoint data and use the average values for the interval associated with "speed". A triangular (or other shape) MF has been constructed whose base endpoints (on the x-axis) are at the two average values and whose apex is midway between the two endpoints. The T1 triangular MF has been represented in two dimensions and expressed mathematically in equation (1)

$\{(\mathrm{x}, \mathrm{MF}(\mathrm{x})) \mid \mathrm{x} \in \mathrm{X}\}$

However, the MF completely ignores the uncertainties associated with the two endpoints. A second approach calculates the average values and the standard deviations for the two endpoints. The approach blurs the location in between the two endpoints along the $\mathrm{x}$-axis. Now the triangles are located in such a way so that their base endpoints can be anywhere in the intervals along the $\mathrm{x}$-axis associated with the blurred average endpoints, which leads to a continuum of triangular MFs on the $\mathrm{x}$-axis. Thus whole bunch of triangles, all having the same apex point but different base points are obtained as shown in figure 5. Suppose, there are exactly $\mathrm{N}$ such triangles, and at each value of $x, M F s$ are: MF1(x), MF2(x), .., MFN(x). Weight is assigned to each membership value, say $w x 1, w x 2, \ldots, w x N$, representing the possibilities associated with each triangle at a particular value of $\mathrm{x}$.

The resulting T2 MF is expressed using (2)

$$
(x,\{(\operatorname{MFi}(x), w x i) \mid i=1, \ldots, N\} \mid x \in X\} \ldots . .(2)
$$

Another way to represent the membership value: $\{(x, \operatorname{MF}(x, w) \mid x \in X$ and $w \in J x\}$ where $\operatorname{MF}(x, w)$ is the threedimensional T2 MF, shown in figure 1. 


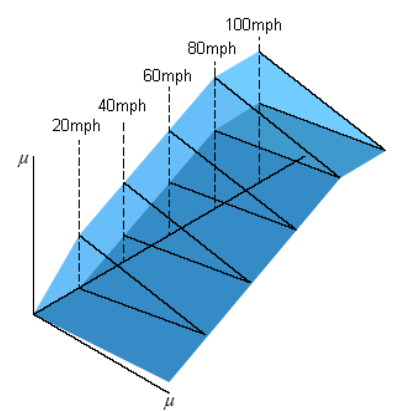

Fig. 1 3-D Representation of T2 FS "Speed"

Another way to visualize T2 fuzzy sets is to plot their footprint of uncertainty (FOU).

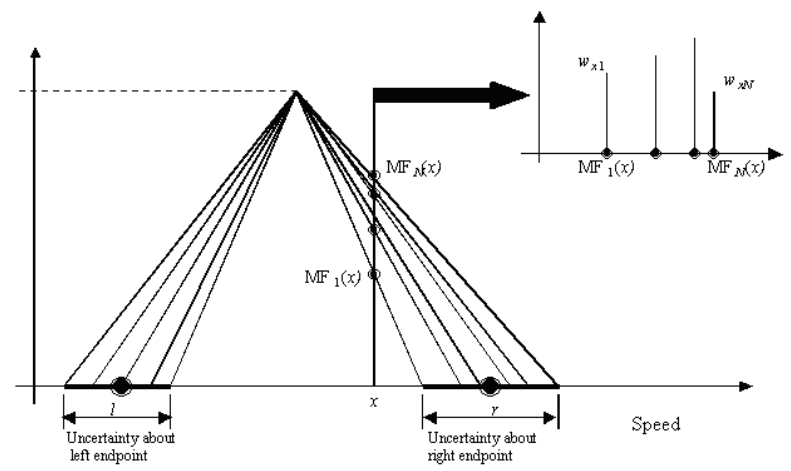

Fig. 2 Triangular MFs (base endpoints 1 and r) with Uncertain Intervals

\section{FOOTPRINT OF UNCERTAINTY}

In $\mathrm{T} 2, \operatorname{MF}(\mathrm{x}, \mathrm{w})$ can be represented in a two-dimensional $\mathrm{x}$-w plane, consisting of only the permissible (sometimes called "admissible") values of $\mathrm{x}$ and $\mathrm{w}$. It implies that $\mathrm{x}$ is defined over a range of values (its domain), say, $\mathrm{X}$ while $\mathrm{w}$ is defined over its range of values (its domain), say, W. An example of FOU for a Gaussian MF is shown. The standard deviation of the MF is certain while mean, $\mathrm{m}$, is uncertain and varies anywhere in the interval from $\mathrm{m} 1$ to $\mathrm{m} 2$. Uncertainty in the primary memberships of a T2 fuzzy set, $\tilde{\mathrm{A}}$, consists of a bounded region, called the footprint of uncertainty (FOU). FOU is the union of all primary memberships $(\mathrm{Jx})$, given in (3).

$\mathrm{FOU}(\tilde{\mathrm{A}})=x \in X$

$$
\bigcup \begin{aligned}
& \bigcup \\
& x
\end{aligned}
$$

FOU focuses our attention on the uncertainties inherent in a specific T2 membership function, whose shape is a direct consequence of the nature of the uncertainty. The region of FOU indicates that there is a distribution that sits on top of it - the new third dimension of T2 fuzzy sets. Shape of the distribution depends on the specific choice made for the secondary grades. When the secondary grade is equal to one, the resulting T2 fuzzy set is called interval T2 fuzzy sets (IT2FS), representing uniform weighting (possibilities).

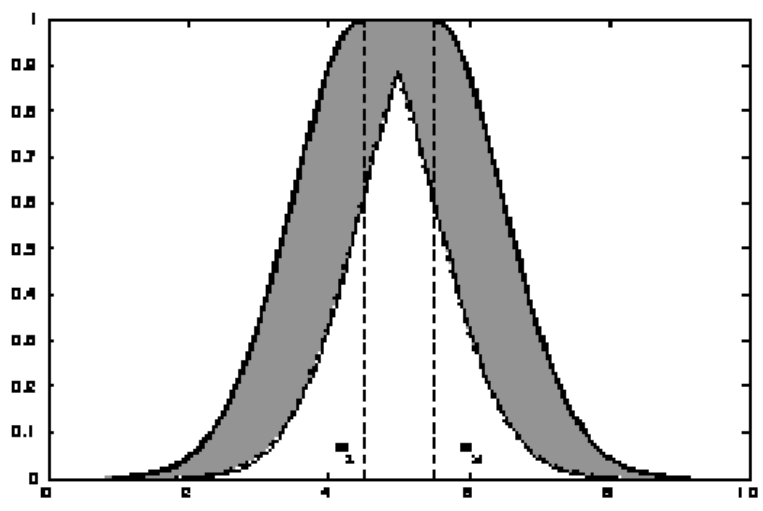

Fig. 3 OU of Gaussian (primary) MF with Uncertain mean 


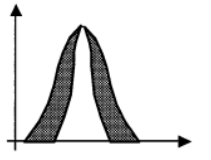

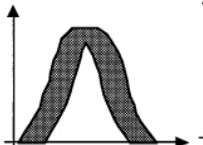

(b)

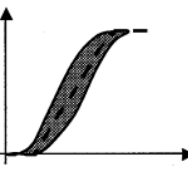

(c)

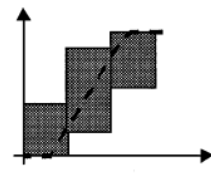

Fig. 4 OU: (a) Gaussian MF with Uncertain Standard Deviation (b) Gaussian MF with uncertain mean (c) Sigmoidal MF with Inflection Uncertainties (d) Granulated Sigmoidal MF with Granulation Uncertainties.

\section{TYPE-2 FUZZY SET ENTROPY}

The process of obtaining necessary information to perform segmentation leads to the correct selection of the regions of interest of the color image. The proposed work applied theory of fuzzy set to evaluate the regions of interest with fixed accuracy. Fuzziness index [12] and entropy [13] provide the measurement of degree of uncertainty [14] of the segmentation process. To measure the fuzziness of images, a few formal definitions are discussed below. An ordinary fuzzy set $\mathrm{A}$ of the universe of discourse $\mathrm{X}$ is classically defined by its membership function $\mu \mathrm{A}(\mathrm{x}): \mathrm{X} \rightarrow[0,1], \mathrm{x} \in \mathrm{X}$.

A point $\mathrm{x}$ for which $\mu \mathrm{A}(\mathrm{x})=0.5$ is said a crossover point of fuzzy set $\mathrm{A} \subseteq X$. The uncertainty is represented by the " $\alpha$-cut" of fuzzy set $A$, whose membership function $\mu \alpha \mathrm{A}(\mathrm{x}): \mathrm{X} \rightarrow\{0,1\}$ is defined in (4).

$\mu \alpha \mathrm{A}(\mathrm{x})=1$ if $\forall \mathrm{x} \geq \alpha$

$$
=0 \text { if } \forall \mathrm{x}<\alpha
$$

Where $\alpha \in[0,1]$ and $\mathrm{x} \in \mathrm{X}$

The fuzziness index $\gamma(\mathrm{A})$ of a fuzzy set $\mathrm{A}$ reflects the degree of ambiguity by measuring the distance $\mathrm{d}(\mathrm{A}, \mathrm{A} 0.5)$ between $\mathrm{A}$ and its nearest set $\mathrm{A} 0.5(\alpha=0.5)$ as described in (5).

$\gamma(\mathrm{A})=2 \times \mathrm{d}(\mathrm{A}, \mathrm{A} 0.5) / \mathrm{n} 1 / \mathrm{p}$

A positive scalar $\mathrm{p}$ is introduced to keep $\gamma(\mathrm{A})$ in between zero and one depending on the type of distance function used. In the proposed algorithm with the help " $\alpha$-cut" " $n$-cut" fuzzy set is described, where $n$ is the number of elements of $n$-cut vector. This measure represents the area between two membership functions $\mu \mathrm{A}(\mathrm{x})$ and $\mu \alpha \mathrm{A}(\mathrm{x})$, described in (6).

$\gamma(\mathrm{A})=\lim _{\Omega \rightarrow X} \frac{1}{\|\Omega\|} * \int_{\Omega}\left|\mu_{A}(x)-\mu_{A}^{0.5}(x)\right| d x$

where $\|\Omega\|$ represents the size of the set $\Omega$ ( linear index values ) and in practice we can use the discrete formula, given in (7)

$$
\gamma_{A}^{p}=\left[\frac{1}{\|X\|} * \sum_{x \in X}\left|\mu_{A}(x)-\mu_{A}^{0.5}(x)\right|^{p}\right]^{\frac{1}{p}}
$$

$\gamma \mathrm{pA}$ is a monotonic function, where $\mathrm{p} \in[1,+\infty]$ and $\|X\|$ represents the cardinality of the set $\mathrm{X}$.

The term entropy of fuzzy set A, denoted by H(A) (monotonic increasing function) was first introduced by De Luca and Termini, expressed in (8).

$$
\begin{gathered}
\mathrm{H}(\mathrm{A})=\left(\sum S_{n}\left(\mu_{A}(x)\right)\right) / n \cdot \ln 2 \\
S_{n}\left(\mu_{A}(x)\right)=-\mu \mathrm{A}(\mathrm{x}) \ln (\mu \mathrm{A}(\mathrm{x}))-(1-(\mu \mathrm{A}(\mathrm{x})) \ln (1-\mu \mathrm{A}(\mathrm{x})))
\end{gathered}
$$

In this work, we use the extension of the "De Luca and Termini" measure to discrete images, proposed by Pal [53]. The (linear) index of fuzziness of an $\mathrm{M} \times \mathrm{N}$ image subset $\mathrm{A} \subseteq \mathrm{X}$ with $\mathrm{L}$ gray levels $g \in[0, \mathrm{~L}-1]$ is defined in (9) and shown in figure 5.

$$
\gamma_{(\mathrm{A})}=\frac{1}{M N} \sum_{g=0}^{L-1} h(g) *\left[\mu_{u}(g)-\mu_{l}(g)\right]
$$

Where $\mathrm{h}(\mathrm{g})$ represents the histogram of the image and $\mu \mathrm{X}(\mathrm{g})$, the membership function consists of $\mu \mathrm{u}(\mathrm{g})$ and $\mu \mathrm{l}(\mathrm{g})$. Entropies are used in with T2 fuzzy sets in gray scale image segmentation by extending the works proposed by Tizhoosh. Tizhoosh applied T2 fuzzy sets for gray scale image thresholding and obtained good results even in case of very noisy images. As proposed in, he used interval T2 fuzzy sets with the FOU, described below:

Upper Limit: $\mu_{u}(x): \mu_{u}(x)=[\mu(\mathrm{x})] 0.5$
Lower Limit: ${ }_{l}(x): \mu_{l}(x)=[\mu(\mathrm{x})] 2$ 


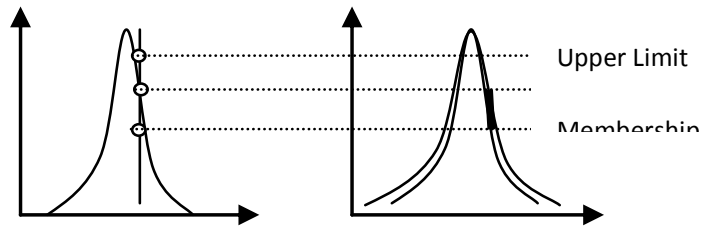

Fig. 5 Membership functions representing FOU

Here for the CMYK color model, the same functions are used for image segmentation. To overcome the drawback of gray scale imaging, various correctional measures are considered in the proposed algorithm.

\section{ALGORITHM FOR COLOR IMAGE SEGMENTATION}

Begin

Step 1:Read the JPEG file to be segmented

Step2:Select the proper shape of the interval base T2 fuzzy set MF as

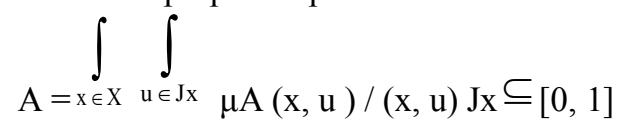

Step3:Fix the image size of $\mathrm{M} \times \mathrm{N}$ matrix;

Step4:Calculate $\mathrm{h}(\mathrm{g})$ for each color component of the color space;

$/ /$ For linear index calculation

Step5:Calculate $\mathrm{n}$ - cut of the total image //for color pattern possibility matching with CMYK;

Step6:Initialize the position of the T2 MF;

Step7:Shift the MF with gray level ranges;

Step8: Mapping the picture colors into gray scale format;//For contour detection

Step 9: calculate the values of $\mathrm{MF} \mu_{u}(g)$

and $\mu_{l}(g)$ (where $\left.\mu \mathrm{u}(\mathrm{g}), \mu \mathrm{l}(\mathrm{g}) \subseteq \mu \mathrm{X}(\mathrm{g})\right)$;

Step10: Compute edge of the image based on

contour formation;

Step11: Compute similarity matrix say ' $\mathrm{W}$ ' based on inverting contours;

Step12: Find mid, max and min of fuzzy index;

Step13: Compute the $\mathrm{n}$ - cut eigenvectors;//possible

combination of colors for the input picture

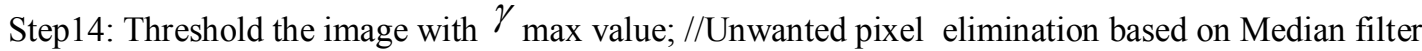

Step15: Masking of segmented image using msk matrix is defined by

$$
\left[\begin{array}{llllllll}
0 & 0 & 0 & 0 & 0
\end{array}\right.
$$

011110

$\mathrm{msk}=\quad 01110$

01110

$\begin{array}{lllllllll}0 & 0 & 0 & 0 & 0\end{array}$

Step16: Median filtering on the segmented image to remove noise;

Step17: Apply the region merging process using the obtained classes of pixels; //Segmented portion of images are merged.

Step18: Smoothing of image to reduce the number of connected components;

Step19: Calculate the connected components;//n-Cut Eigen vector

Step20: Calculate the number of pixels in the final image;

End.

XII. EXPERIMENTAL RESULTS

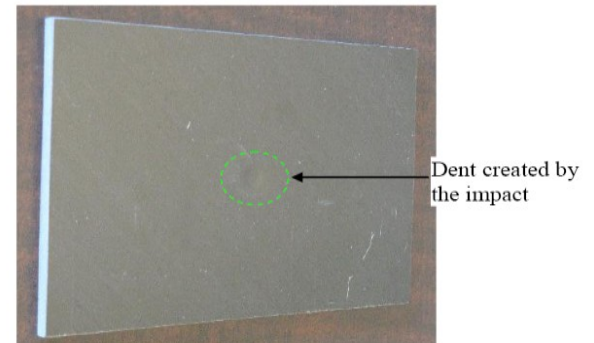

Fig 6: A photograph of an impacted sample. 
In most papers available in the open literature, signal processing is applied to simple geometry simulated flaws such as square Teflon ${ }^{\circledR}$ inserts or flat bottom holes. Example of typical results obtained by processing PT inspection results of a composite sample that contains flat bottom holes are shown in Figure 7.

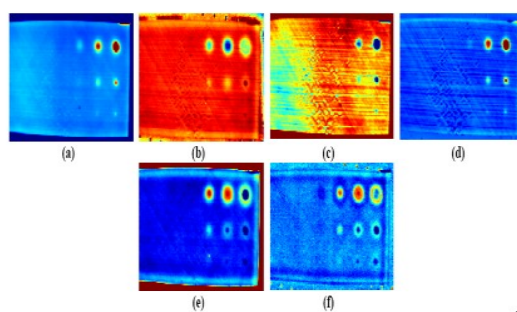

Fig 7: Typical images of (a) amplitude, (b) phase, (c) 1 st principal component, (d) $2^{\text {nd }}$ principal component (e) first derivative and (f) second derivative of a sample containing flat bottom holes.

As seen in the images presented in Figure 2, typically, except for the edge effect18, the flaws exhibit a uniform feature. The reason being that artificial flaws used in laboratory samples are usually at one specific depth. The reality is that a flaw can occur at different depths. For example an impact can cause fiber and matrix breakage resulting in a surface dent while creating delamination damage in several deeper layers. In addition, real components might be painted, be covered by a label or have information written on them that can affect the thermogram and make automated detection challenging. Examples of processed pulsed thermography results of impact-induced damage are presented in Figures 8 and 9.

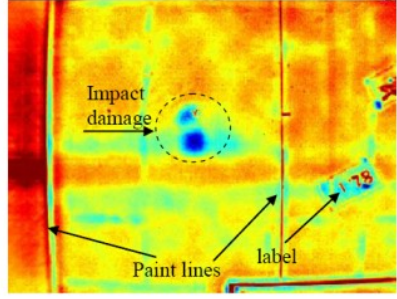

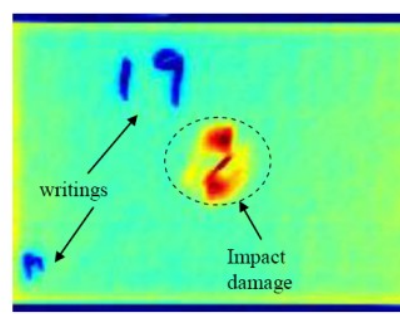

(b)

Fig 8: Pulsed thermography images of different samples containing impact damage, paint lines, sticker and marker writings.
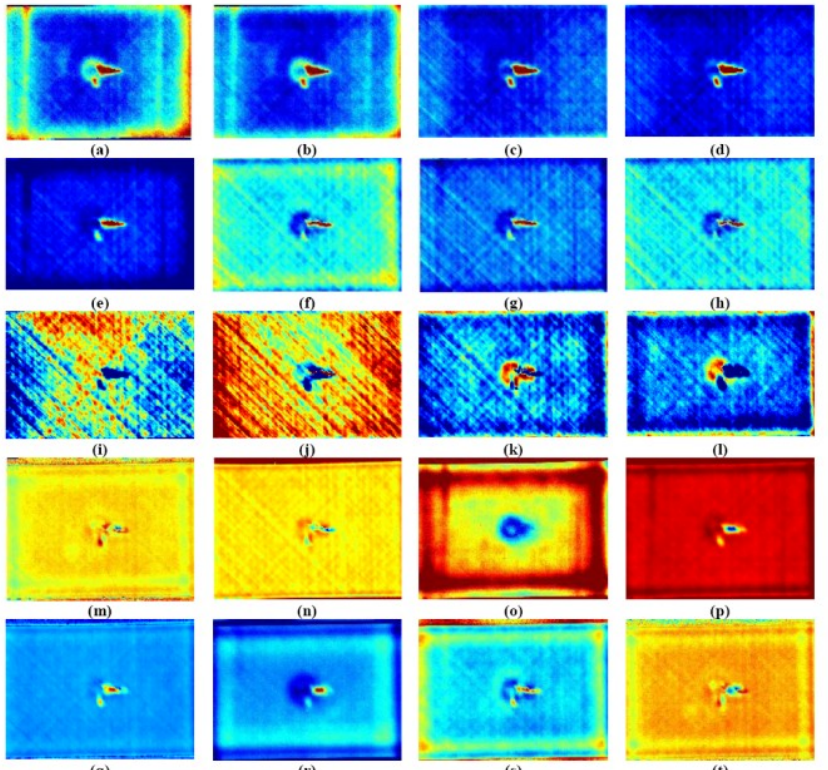
Fig 9: First four non-null images from (a-d) amplitude; (e-h) phase; and (i-1) principal components; and examples of 1st (m-p) and 2nd (q-t)
derivative images of the same sample.

Although simply applying a threshold on the data obtained on laboratory samples can yield good results for automated detection, it lacks the robustness required to deal with real damage. Besides, due to the edge effect it can either underestimate or overestimate the flaw size. In addition, real damage does not behave like simulated flaws and sizing techniques such as the full-width half-maximum cannot be applied easily to estimate the flaw size. As it can be seen in Figure 4, amplitude, phase and principal component images provide different information and flaw sizes. One of the challenges is to select the proper images to accurately estimate the flaw size. The amplitude, phase and principal component images tend to show the flaw within the first few images; 
while derivative processing algorithms require browsing through more images in the sequence to see the entire flaw. Therefore the former techniques were selected to develop the proposed algorithm. The first step of the algorithm proposed is to compute the amplitude, phase and principal component images. Then, the marking and writing are automatically detected and removed from the images by creating a mask. For the samples used, it was found that the writing of the last amplitude image had values less than two times the standard deviation (STD) compared to the average of the panel.

Then the selected images: amplitude images 2 to 6 , phase images 2 to 10, and PCA images 1 to 6 were further processed. For each of the selected amplitude images, each pixel that had a value of one STD above the average was considered being part of a flaw. Similarly, for the selected phase and PCA images each pixel that had a value of one STD above or below the average of the sample was considered to be a flaw. This resulted into several binary images that were summed up to give a "flaw likelihood" image as shown in Figure 10(a). After summation, each pixel that had a value superior to 4 was considered a flaw. An example of a binary image obtained after applying the threshold to the flaw likelihood image is presented in Figures 10(b). A series of morphological filters were then applied to remove what is considered to be noise and filling holes in such a way that only the large connected components of binary image were kept, as shown in Figure 11 (a). The agglomeration of pixels corresponding to the damage area was identified to be the one which had the highest pixel value in the second amplitude image (Figure 11 (b)).
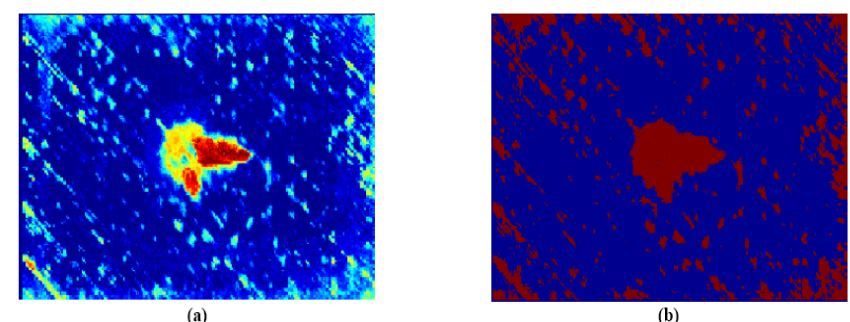

Fig 10: An example of a (a) damage likelihood image (b) flaw image after applying threshold.

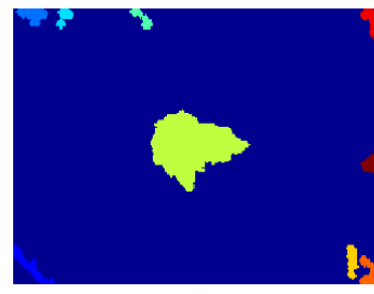

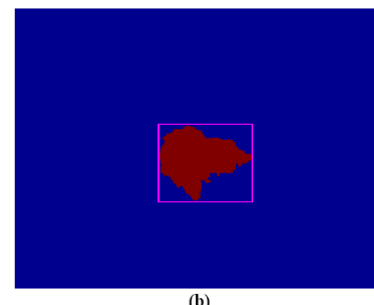

Figure 11: An example of a (a) flaw image after applying morphological operations (b) flaw image after identifying the image blob corresponding to the damage area.

The inspection results from both the front and the back of the samples were processed using this algorithm. In each case the algorithm successfully identified the damaged area and the false calls rate was zero. The final flaw image obtained (Figure 11 (b)) was used to calculate the flaw size and area. These measurements were similar to those obtained by an inspector measuring the flaw based on the regular processing techniques presented in section 2. The measurements obtained were then compared to those obtained by pulse-echo ultrasonic. It was found that on average the measurements by thermography underestimate the flaw width and length by $15 \%$ and $10 \%$ respectively. Since the measurements obtained by the automated algorithm are similar to those obtained by an inspector, the differences may be caused by the samples geometries and its material characteristic. Further trial of the algorithm on different sample geometries should be carried out to better explain these differences.

Although the process of creating this image is more computation intensive than other techniques used individually, it allows simplifying an entire thermogram sequence into a single image. Pulsed phase thermography and PCA significantly reduces the number of images to analysis, but combining these techniques reduces it even further.

And in order to test the performance of the algorithm, the samples (figure 12 onwards) are taken in JPEG image format having a size of $163 \times 147$ pixels in RGB color mode. Results of execution of the algorithm are shown from figure 12 . 


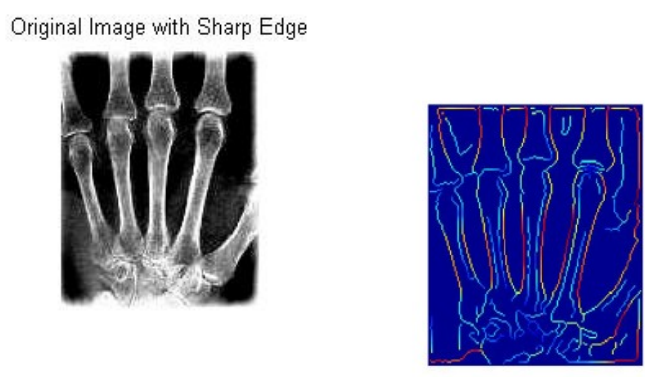

Fig. 12 Original image

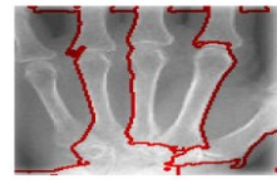

Fig. 13 Segmented Image

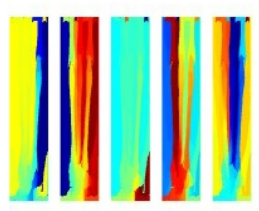

Fig. 14 Contour Detection

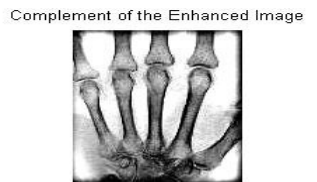

Fig. 15 n-cut eigenvectors

Segmentation of the main image

!II!!

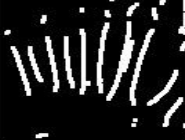

\section{A. Screenshot of matlab}

Fig. 16 Complement of the Enhanced Image

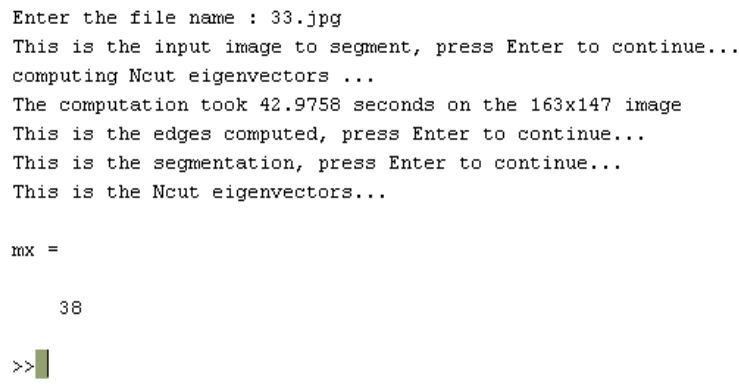

\section{CONCLUSION}

An algorithm was developed for automated flaw detection and measurement of pulsed thermography inspection data. The algorithm was based on the combination of information from typical signal processing techniques used in pulsed thermography. The statistical divergence of the pixel value compared to the sample allowed identifying the damaged area. The algorithm provided measurements similar to that of a human eye. 
Moreover, the image obtained during the processing was easier to interpret by the inspector, provided the damage area with stronger contrast compared to other processing techniques used individually.

Also Medical image registration has been an important area of research in the medical application of computer vision's techniques for the past several years. It can be defined as a task of finding the transformation that will optimally superimposes features from one imaging study over those of another study. The rules were applied to predict the transient flow and pressure distributions in the brain vasculature comprising a patient specific circle of Willis geometry and fractal models of peripheral vascular networks. The rules were shown to be able to efficiently provide detailed descriptions of the flow and pressure distributions at different levels of blood vessel sizes and simulate the variations of the blood flow in the major cerebral arteries when the peripheral vasculatures are subjected to various physiological and pathological conditions. In order to improve the prediction, the mechanisms of active regulation of blood flow need to be defined and implemented in the future model development.

\section{REFERENCES}

[1] Maldague Xavier, "Theory and Practice of Infrared Technology for Nondestructive Testing”, John Wiley \& Sons Inc, New York NY, pp. 684, 2001

[2] D. Gonzalez, Clemente Ibarra-Castanedo, F.J. Madruga and Xavier Maldague, "Differentiated Absolute Phase Contrast Algorithm for the Analysis of Pulsed Thermographic Sequences", Infrared Physics and Technology, Volume 48, Issue 1, pp. 16-21, 2006.

[3] Mirela Susa, Hernan Dario Benitez, Clemente Ibarra-Castanedo, Humberto Loaiza, Abdel Hakim Bendada and Xavier Maldague, "Phase contrast using differential absolute contrast method", QIRT (Quantitative InfraRed Thermography) Journal, vol. 3, no 2, pp. 219- 230, 2006.

[4] Hernan Dario Benitez, Clemente Ibarra- Castanedo, Abdel Hakim Bendada, Xavier Maldague, Humberto Loaiza and Eduardo Caicedo, "Modified Differential Absolute Contrast Using Thermal Quadrupoles for the Nondestructive Testing of Finite Thickness Specimens by Infrared Thermography", in CCECE 2006 - Canadian Conference on Electrical and Computer Engineering, (Ottawa (Ontario) Canada), May 7-10 2006

[5] Maldague X. and Marinetti S., "Pulse Phase Infrared Thermography”, J.Appl. Phys., Vol. 79, pp. $2694-2698,1996$.

[6] Ibarra-Castanedo C. "Quantitative subsurface defect evaluation by pulsed phase thermography: depth retrieval with the phase," Ph. D. thesis, Laval University, 2005, http://www.theses.ulaval.ca/2005/23016/23016.pdf.

[7] Ibarra-Castanedo, C. and Maldague, X. "Interactive methodology for optimized defect characterization by quantitative pulsed phase thermography," Research in Nondestructive Evaluation, Vol. 16, No. 4, pp1-19, 2005.

[8] Shepard S. M. “Advances in Pulsed Thermography”, Andres E. Rozlosnik, Ralph B. Dinwiddie (eds.), Proc. SPIE, Thermosense XXIII, Vol. 4360, pp. 511-515, 2001.

[9] Carslaw, H. S. and Jaeger, J. C., "Conduction of Heat in Solids", 2nd edition, Clarendon Press, Oxford.

[10] Martin R. E., Gyekenyesi A. L., Shepard S. M., "Interpreting the Results, of Pulsed Thermography Data," Materials Evaluation, Vol. 61, No. 5, pp 611-616, 2003.

[11] Shepard, S. M, "Temporal Noise Reduction, compression and Analysisn of Thermographic Image Data Sequence", US Patent 6,516,084, February, 2003.

[12] Shepard, S. M., Ahmed, T., Rubadeux, B. A., Wang, D., Lhota, J. R., "Synthetic Processing of Pulsed Thermography Data for Inspection of Turbine Components", Insight Vol.43 No.9, September, 2001.

[13] S. Marinetti, E. Grinzato, P.G. Bison, E. Bozzi, M. Chimenti, G. Pieri, O. Salvetti, "Statistical analysis of IR thermographic sequences by PCA", Infrared Physics and Technology 46 (2004) 85-91.

[14] Rajic N., "Principal component thermography for flaw contrastenhancement and flaw depth characterisation in composite structures", Composite Structures 58, pp521-528, 2002.

[15] J.N. Zalameda, P.A. Howell and W.P. Winfree, "Compression Technique Computational Performance," Proceedings of SPIE Thermosense XXVII, Vol. 5782, pp. 399-406, 2005.

[16] K. E. Cramer and W. P. Winfree, "The Application of Principal Component Analysis Using Fixed Eigenvectors to the Infrared Thermographic Inspection of the Space Shuttle Thermal.

[17] Fred L. Bookstein, Principal warps: Thin-plate splines and the decomposition of deformations, IEEE Transactions on Pattern Analysis and Machine Intelligence 11 (1989), no. 6, 567-585.

[18] F. Brezzi and M. Fortin, Mixed and hybrid finite element methods, Springer, 1991.

[19] Morten Bro-Nielsen, Medical image registration and surgery simulation, Ph.D. thesis, MM, Technical University of Denmark, 1996.

[20] Chaim Broit, Optimal registration of deformed images, Ph.D. thesis, Computer and Information Science, Uni Pensylvania, 1981.

[21] Gary E. Christensen and H. J. Johnson, Consistent image registration, IEEE Transaction on Medical Imaging 20 (2001), no. 7, 568582 .

[22] Gary Edward Christensen, Deformable shape models for anatomy, Ph.D. thesis, Sever Institute of Technology, Washington University, 1994.

[23] A. Collignon, A. Vandermeulen, P. Suetens, and G. Marchal, 3d multimodality medical image registration based on information theory, Kluwer Academic Publishers: Computational Imaging and Vision 3 (1995), 263-274.

[24] R. Courant and David Hilbert, Methods of mathematical physiks, vol. II, Wiley, New York, 1962.

[25] Bernd Fischer and Jan Modersitzki, Fast inversion of matrices arising in image processing, Num. Algo. 22 (1999), 1-11.

[26] Fast diffusion registration, AMS Contemporary Mathematics, Inverse Problems, Image Analysis, and Medical Imaging, vol. 313, 2002, pp. 117-129.

[27] Combination of automatic non-rigid and landmark based registration: the best of both worlds, Medical Imaging 2003: Image Processing (J.M. Fitzpatrick M. Sonka, ed.), Proceedings of the SPIE 5032, 2003, pp. 1037-1048. 